\title{
Systematic review of operative outcomes of robotic surgical procedures performed with endoscopic linear staplers or robotic staplers
}

\author{
Mario Gutierrez ${ }^{1} \cdot$ Richard Ditto $^{1}$ (1) $\cdot$ Sanjoy Roy ${ }^{1}$
}

Received: 26 February 2018 / Accepted: 30 April 2018 / Published online: 9 May 2018

(c) The Author(s) 2018

\begin{abstract}
A comprehensive review of operative outcomes of robotic surgical procedures performed with the da Vinci robotic system using either endoscopic linear staplers (ELS) or robotic staplers is not available in the published literature. We conducted a literature search to identify publications of robotic surgical procedures in all specialties performed with either ELS or robotic staplers. Twenty-nine manuscripts and six abstracts with relevant information on operative outcomes published from January 2011 to September 2017 were identified. Given the relatively recent market release of robotic staplers in 2014, comparative perioperative clinical outcomes data on the performance of ELS vs. robotic staplers in robotic surgery is very sparse in the published literature. Only three comparative studies of surgeries with the da Vinci robotic system plus ELS vs. da Vinci plus robotic staplers were identified; two in robotic colorectal surgery and the other in robotic gastric bypass surgery. These comparative studies illustrate some nuances in device design and usability, which may impact outcomes and cost, and therefore may be important to consider when selecting the appropriate stapling technologies/technique for different robotic surgeries. Comparative perioperative data on the use of ELS vs. robotic staplers in robotic surgery is scarce (three studies), and current literature identifies both types of devices as safe and effective. Given the longer clinical history of ELS and its relatively more robust evidence base, there may be trade-offs to consider before switching to robotic staplers in certain robotic procedures. However, this literature review may serve as an initial reference for future research.
\end{abstract}

Keywords Robotic surgical procedures $\cdot$ Surgical staplers $\cdot$ Surgical stapling $\cdot$ Colorectal surgery $\cdot$ Gastric bypass

\section{Introduction}

Stapling is a critical step during many surgical procedures involving the transection of vessels as well as other types of tissue-irrespective of the surgical approach. Staple line integrity is critical to creating a functional anastomosis or a clean transection and has been the focus of continuing innovation by surgical stapler manufacturers [1]. Staple line failure resulting in postoperative leaks is one of the most serious and feared complications for any surgery. Technical aspects of stapling may vary and factors such as anatomical location, tissue viscosity, staple height, and other intrinsic properties of the stapling system itself may substantially influence appropriate staple line formation [2]. Many studies

Richard Ditto

rditto@its.jnj.com

1 Ethicon, Inc., Cincinnati, OH, USA acknowledge that surgeon experience is critical in creating an anastomosis with sufficient staple line integrity to resist leakage and promote healing [3-5].

In most robotic surgical procedures performed in the last decade, the portion of the procedure requiring tissue stapling has been performed by a bedside surgeon/assistant using conventional endoscopic linear staplers (ELS). Starting from the initial mechanically actuated devices, innovation in endoscopic stapling technology has introduced powered devices (available since 2010), which utilize a motor for both staple firing and knife blade action.

In late 2014, Intuitive Surgical (Sunnyvale, CA, USA) received United States Food and Drug Administration (FDA) clearance for the EndoWrist $\mathrm{Xi}^{\circledR}$ stapler (referred to as the EndoWrist Stapling System-EWSS) compatible with the da Vinci Xi Surgical System, which offered the first integrated stapling option for the da Vinci robotic system. This newly integrated stapler allows for the entire procedure to be completed by the console surgeon. Since then, Intuitive Surgical 
has implemented some voluntary recalls and product corrections [6]. Of the 26 Intuitive Surgical EndoWrist Class 2 product recalls documented in the FDA database, 16 (62\%) involve the EWSS [6]. These device recalls suggest that transitioning from ELS to robot-integrated staplers may involve some trade-offs that should be considered before transitioning from ELS to totally robot-integrated staplers. We carried out a review of the literature to assess and summarize reports of operative outcomes of stapled robotic surgical procedures, so that it may serve as a reference for future outcome comparisons of procedures performed with these stapling devices.

\section{Methods}

A systematic literature search of Ovid Embase/Medline, PubMed, and QUOSA was conducted for reports on the topic of robotic surgical procedures performed using ELS published between January 1, 2004 and March 13, 2017. Search keywords included, but were not limited to: robot (and variations like robotic surgery, robot-assisted surgery, robotic surgery), da Vinci (with variations), laparoscopic (with variations), and Echelon, EndoPath, Endo GIA, EndoWrist, stapler (with variations), surgical stapling, endoscopic stapler, linear stapler, flex stapler, endocutter (with variations), endostapler, Ethicon, Covidien, Intuitive. Duplicate publications and preclinical (animal and bench testing) publications were removed. Two investigators reviewed and screened the abstracts of identified studies for relevance and potential inclusion in the review. Pertinent human studies, restricted to the English language were selected for full paper review. Studies were excluded if they did not use stapling during the robotic surgical procedure (e.g., suturing), used a circular stapler only, or if the stapler or robotic system used in the surgical procedure was not specified. Only reports on da Vinci robotic surgical procedures performed using Echelon Flex $^{\mathrm{TM}}$ staplers (Ethicon, Johnson \& Johnson, New Brunswick, NJ) or the Endo GIA ${ }^{\mathrm{TM}}$ staplers (Covidien, Mansfield, MA) and/or EndoWrist $\mathrm{Xi}^{\circledR}$ robotic staplers (Intuitive Surgical, Sunnyvale, CA, USa) were selected for inclusion in this review. The literature search was completed on March 21, 2017 and a weekly alert was set up on QUOSA for relevant key words to continue to identify reports throughout 2017 (referred to here as the manual search).

\section{Results of the literature search}

There were 239 total publications (94 manuscripts and 146 abstracts) identified with potentially relevant information. From the systematic search, 27 manuscripts and 2 abstracts were identified with information directly relevant to this review. Three manuscripts and four abstracts, which were additionally identified from the manual search performed between March 21, 2017 and January 30, 2018, were also included in this review. Thus, the total number of studies included in this review was 36 [7-42]. Figure 1 shows the process of publication selection. The perioperative outcomes reported in the studies are presented in Table 1.

Fig. 1 Publication selection

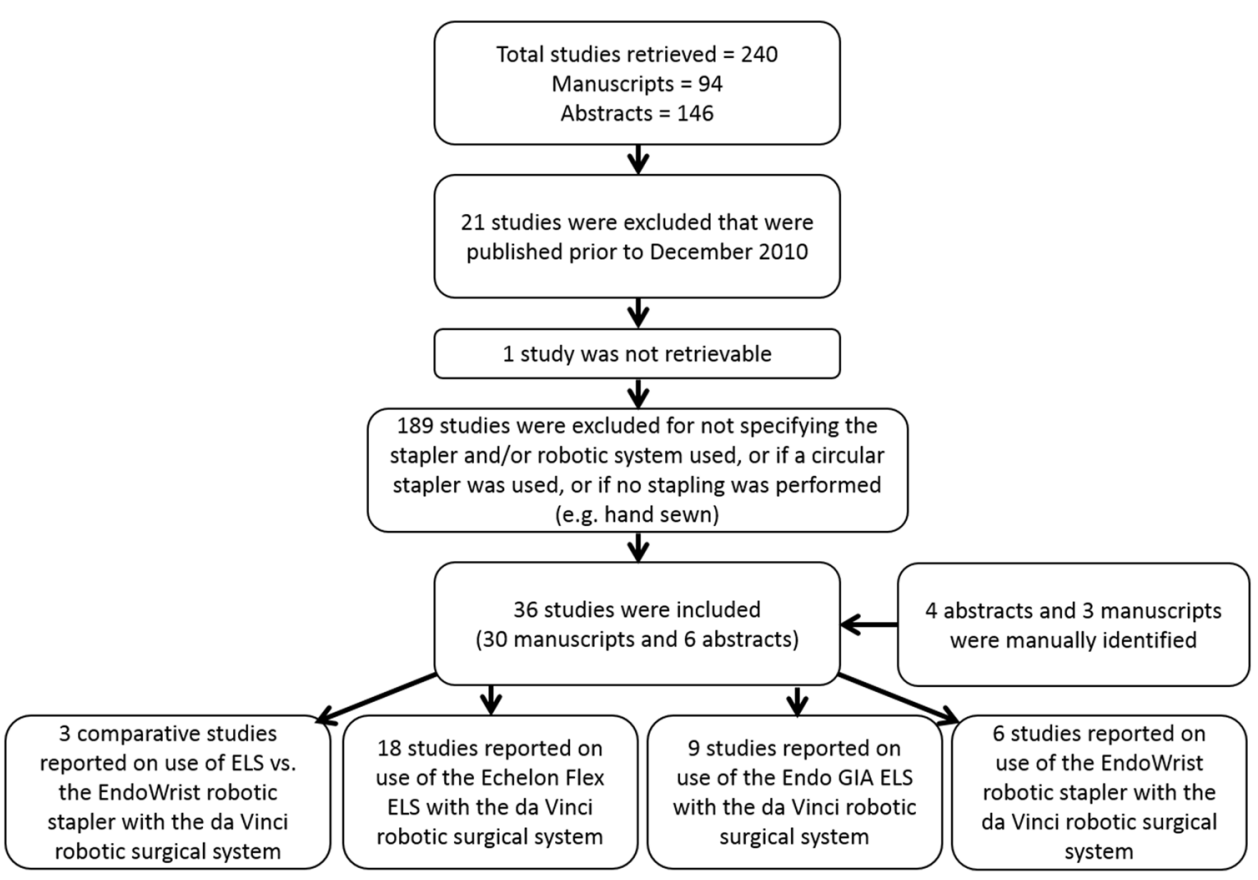




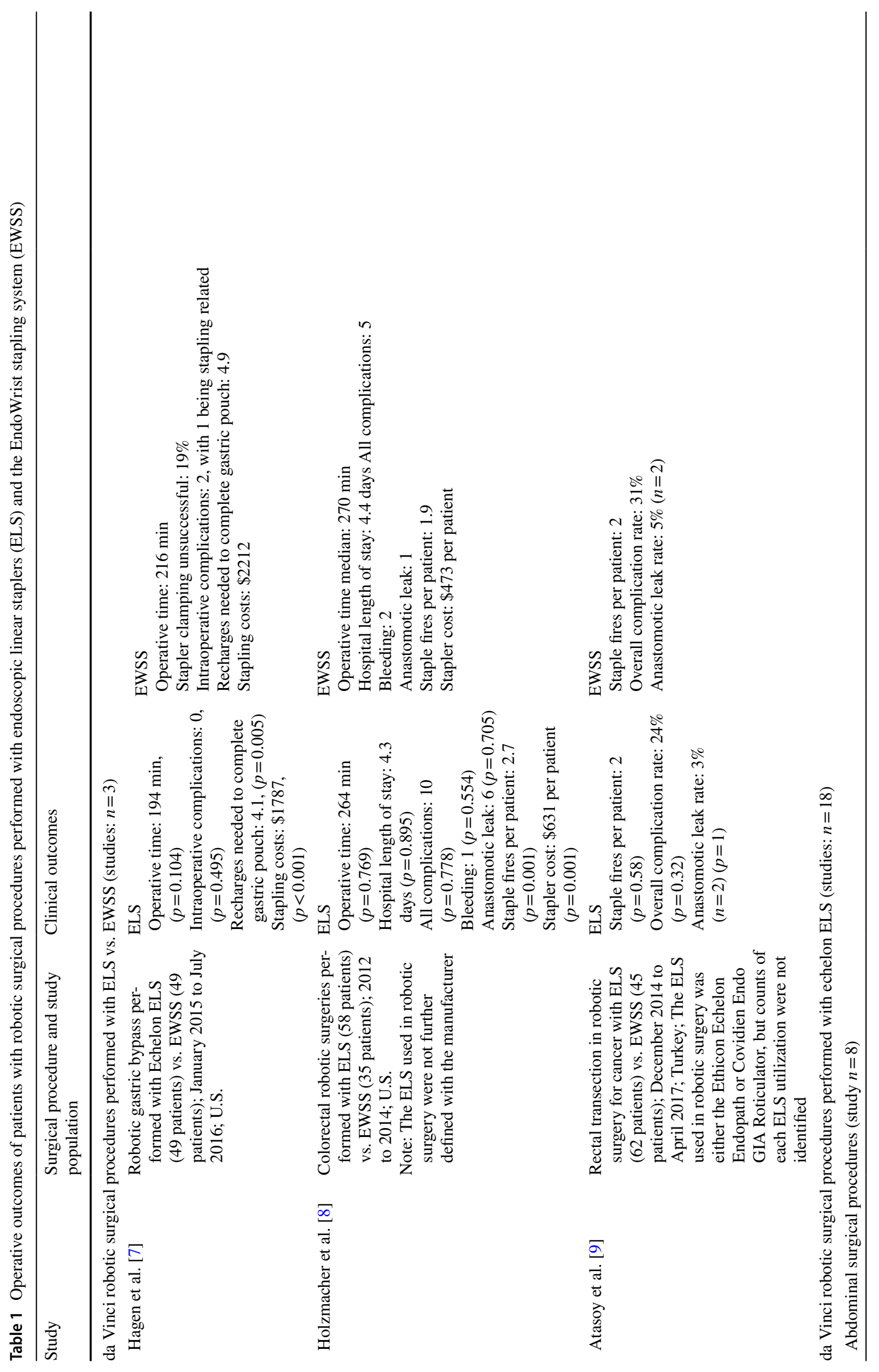




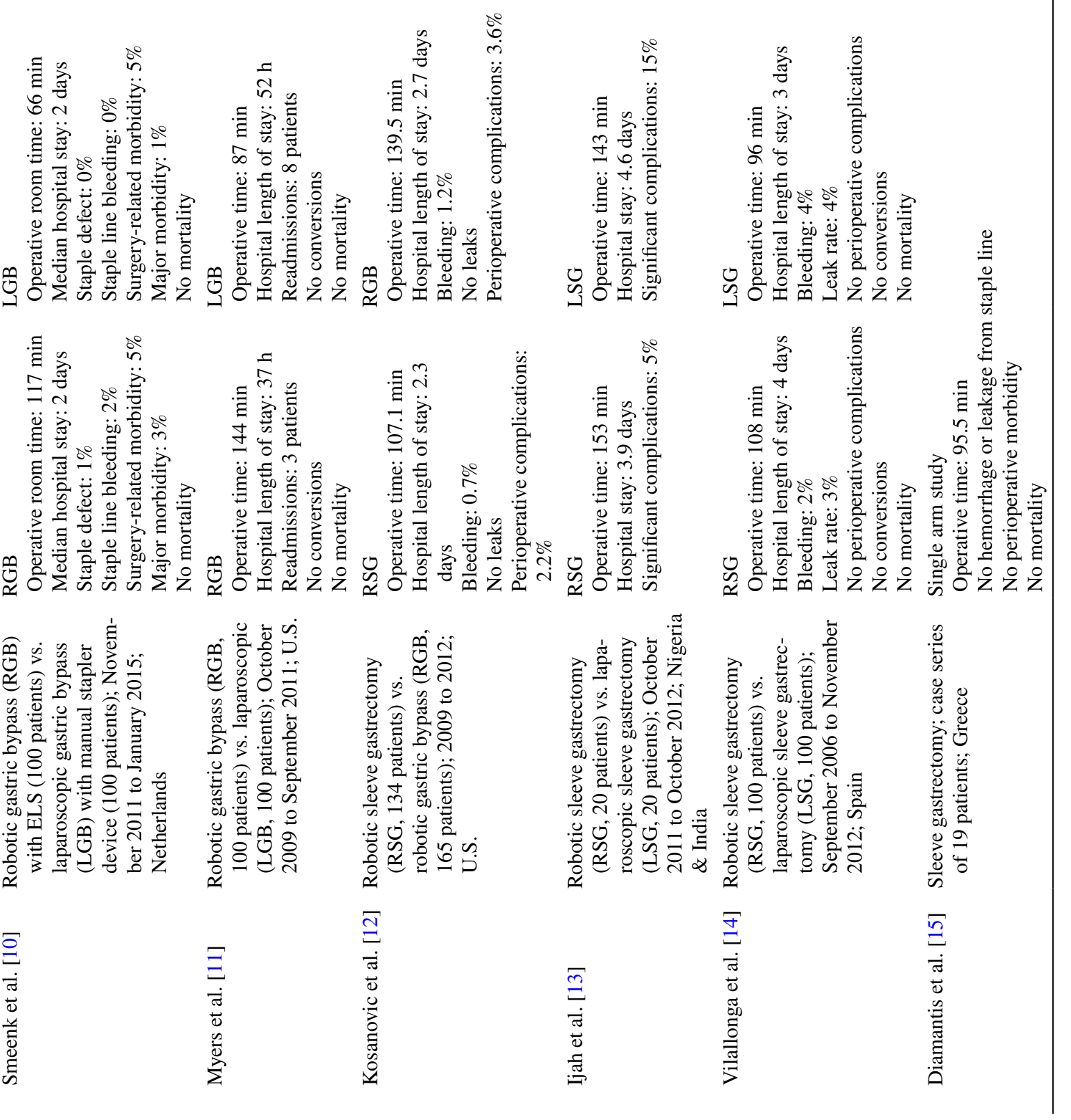




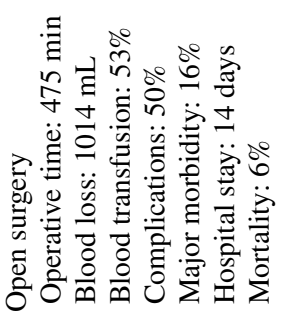

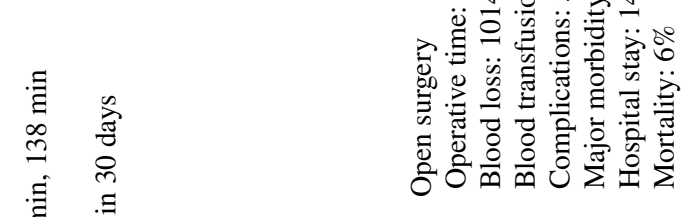

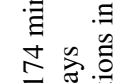

夆

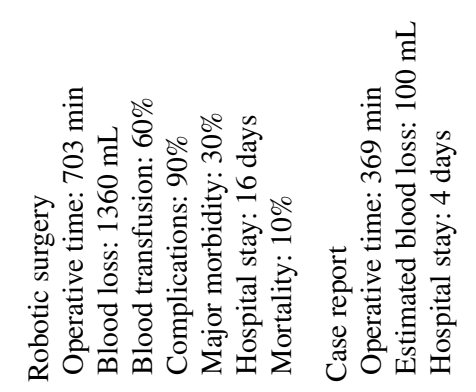

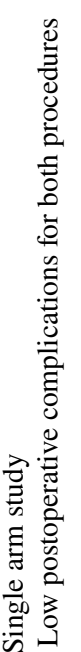

2
8
2

青瓷

要

范苍屍

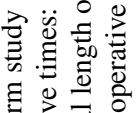

ำ 는

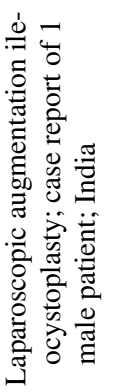

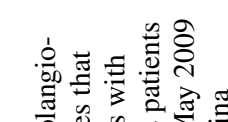

疍.

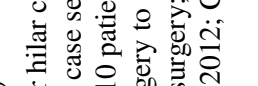

तิ

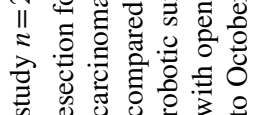

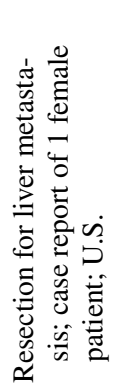

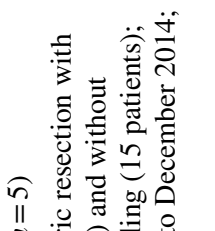

II.

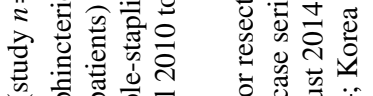

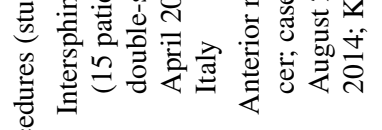

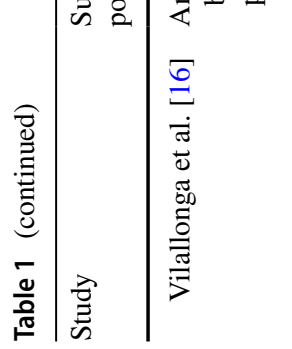

至

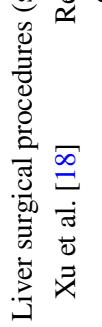

$\simeq$

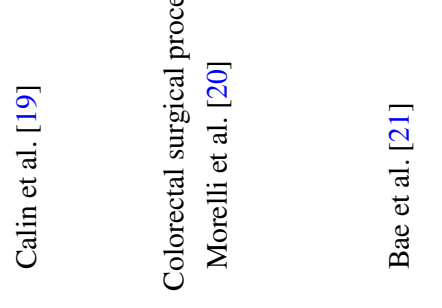

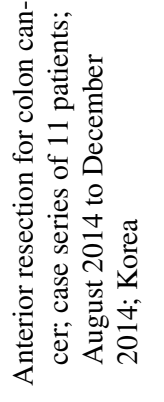




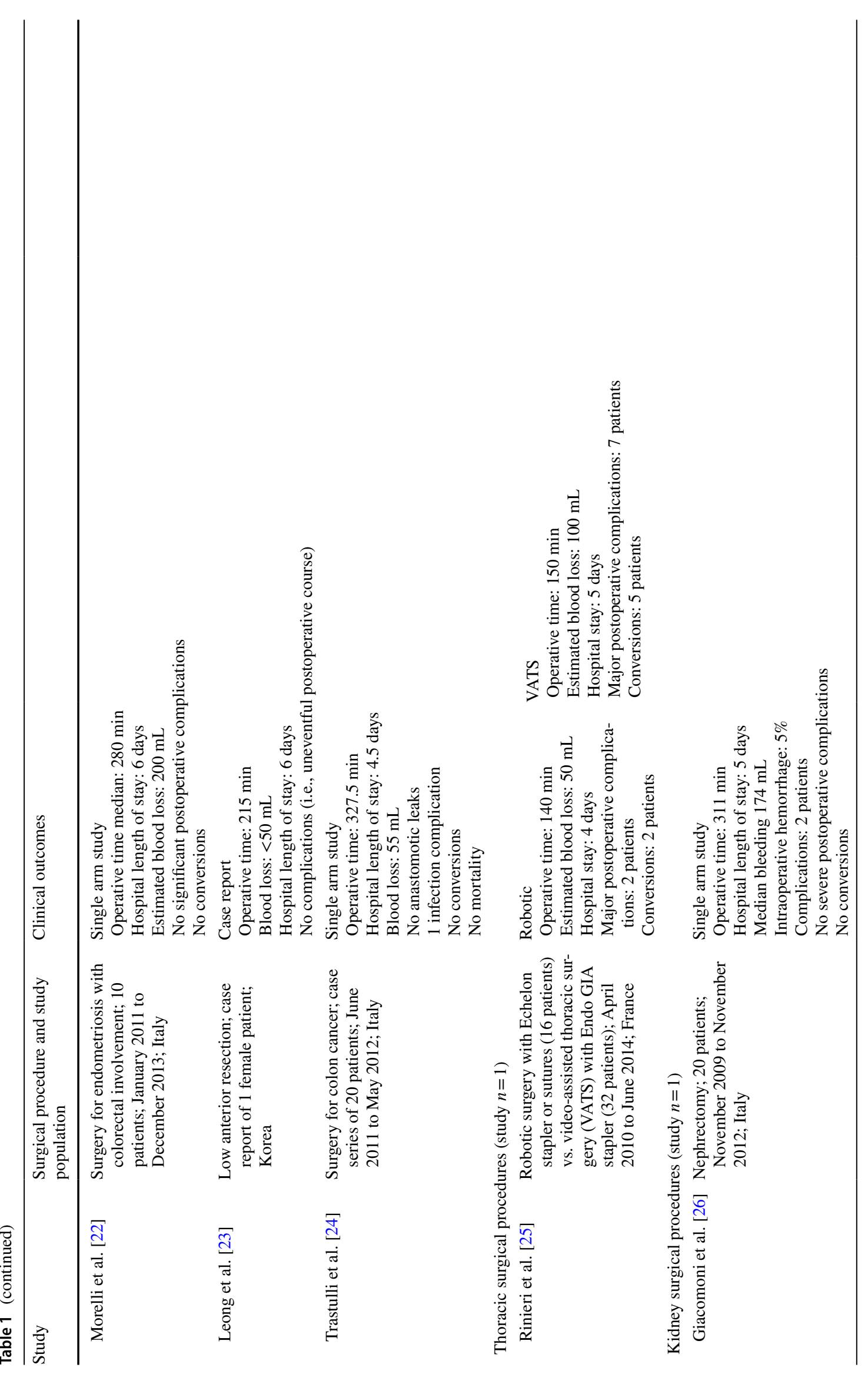



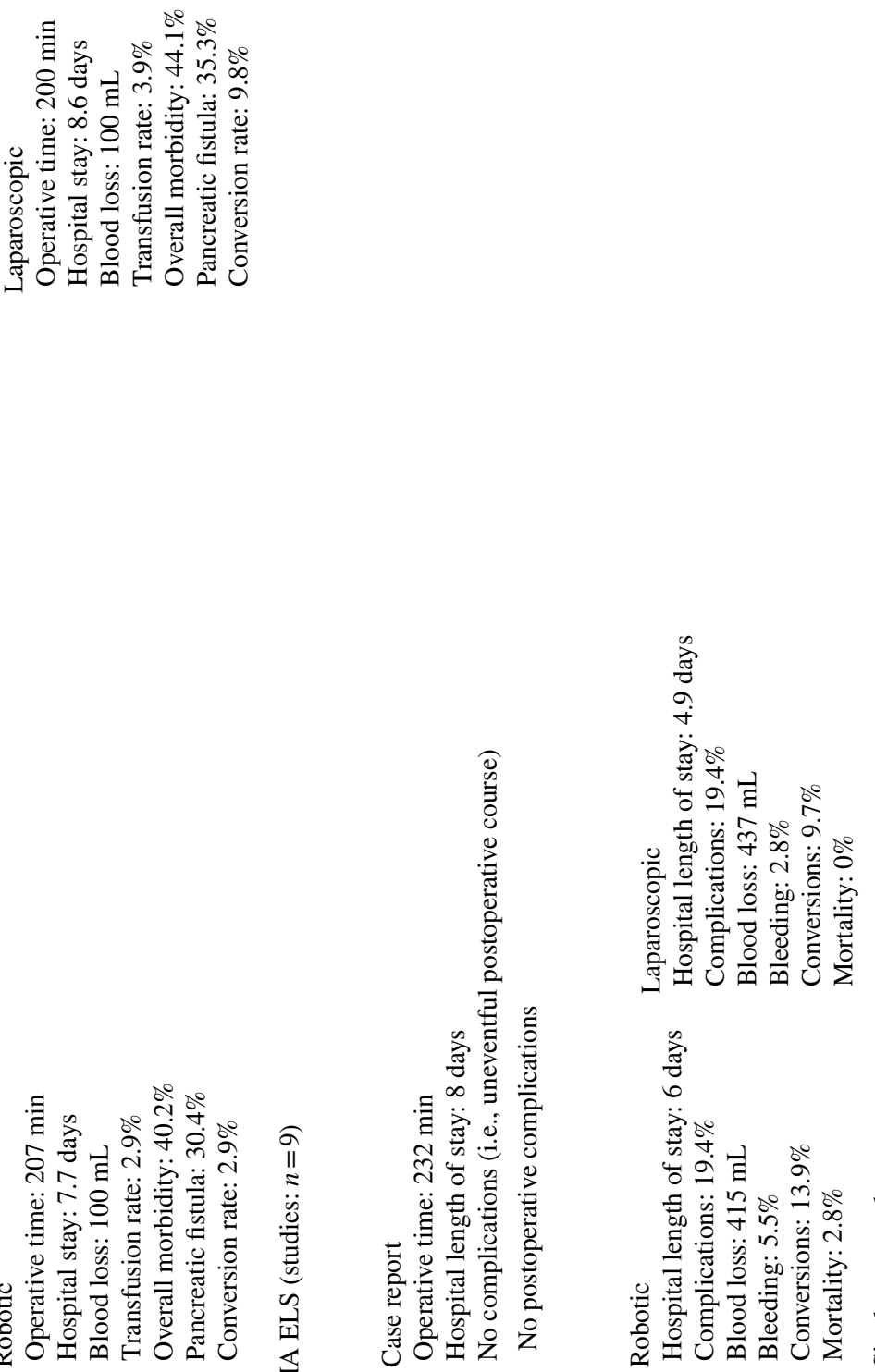

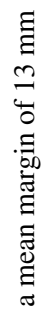

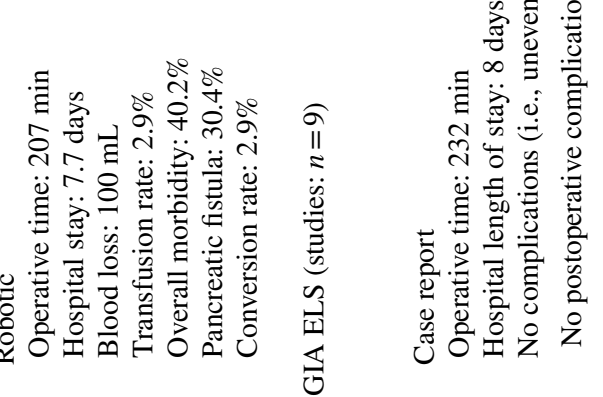

స్

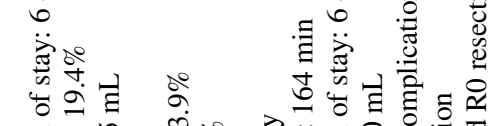

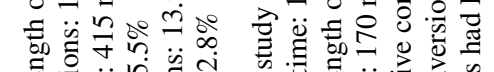

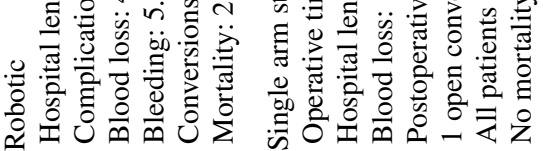

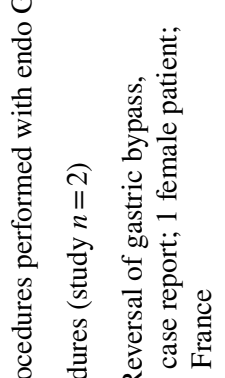

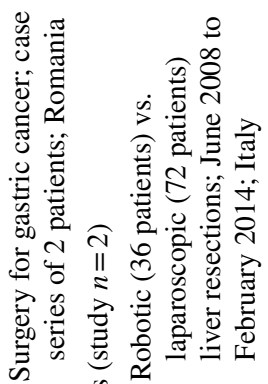

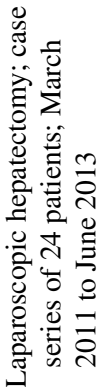

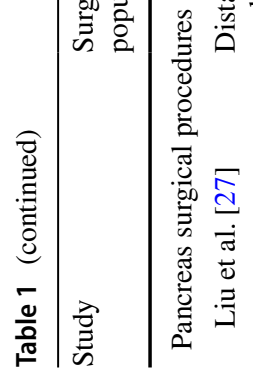

范

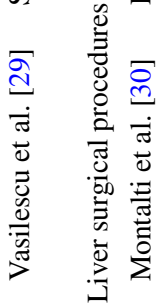

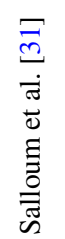




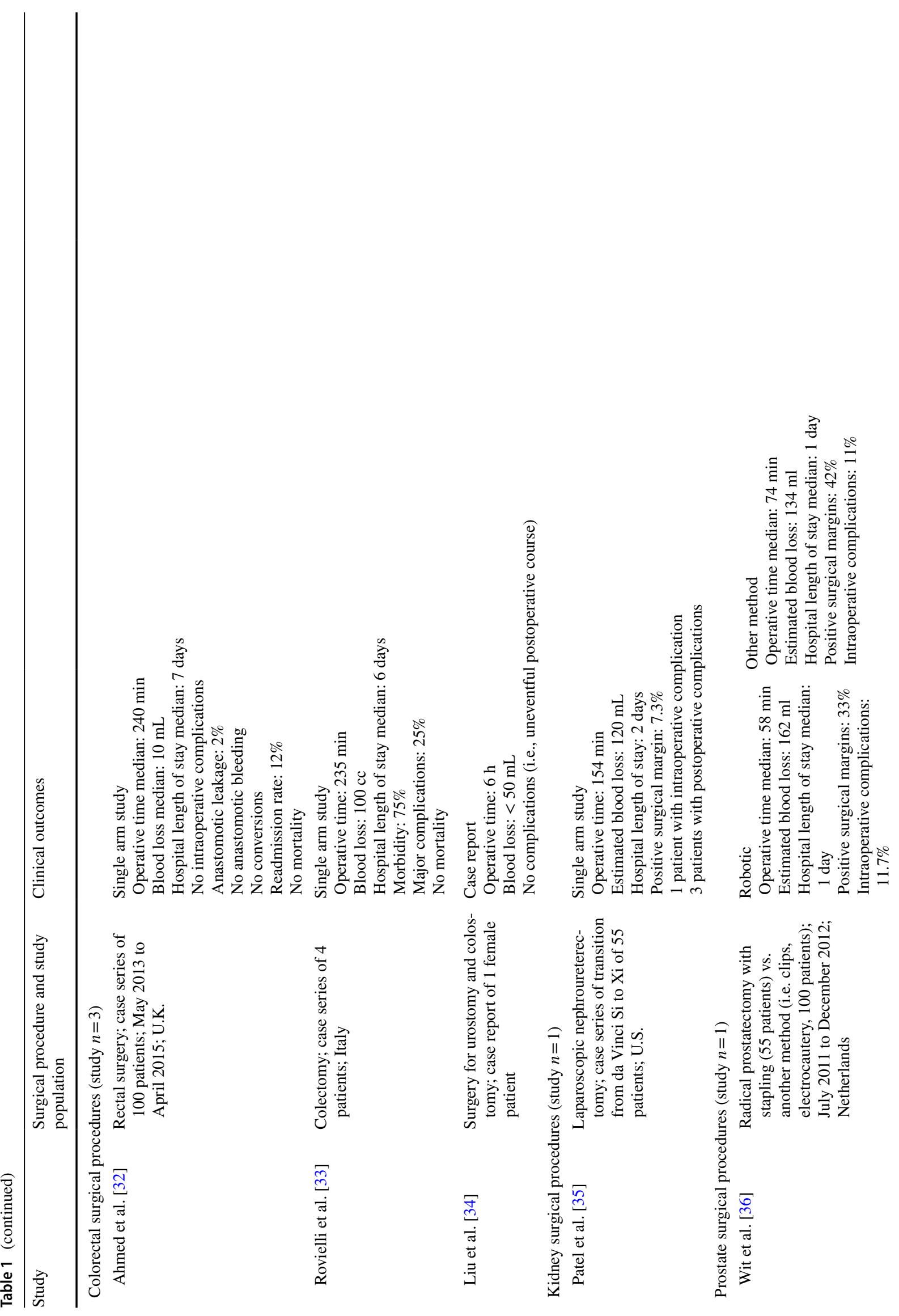




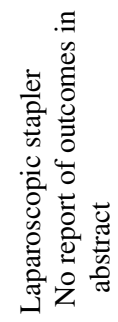

0
0
0
0
0
0
0
0
0
0
0
0
0
0
0
0
0

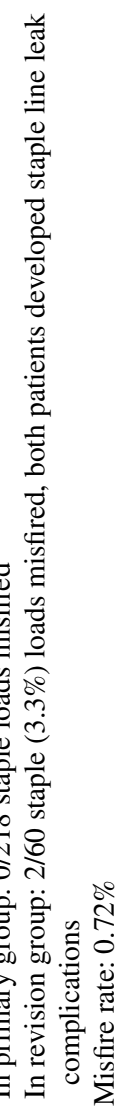

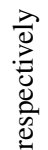

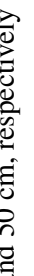

$\vec{m}$

.0

.

竞

莽

竞弯

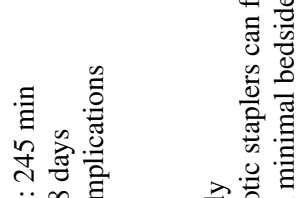

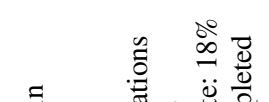

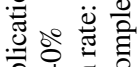

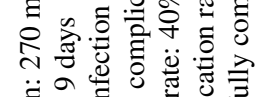

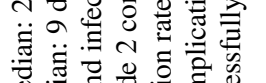

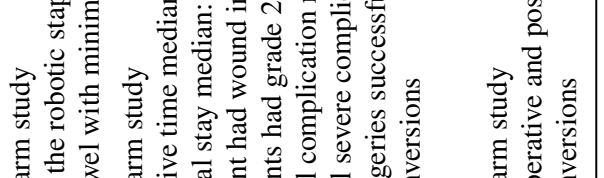

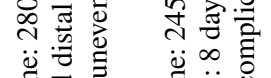

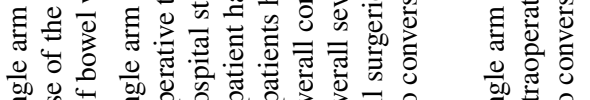

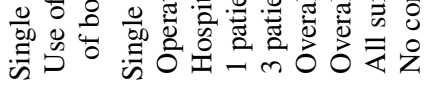
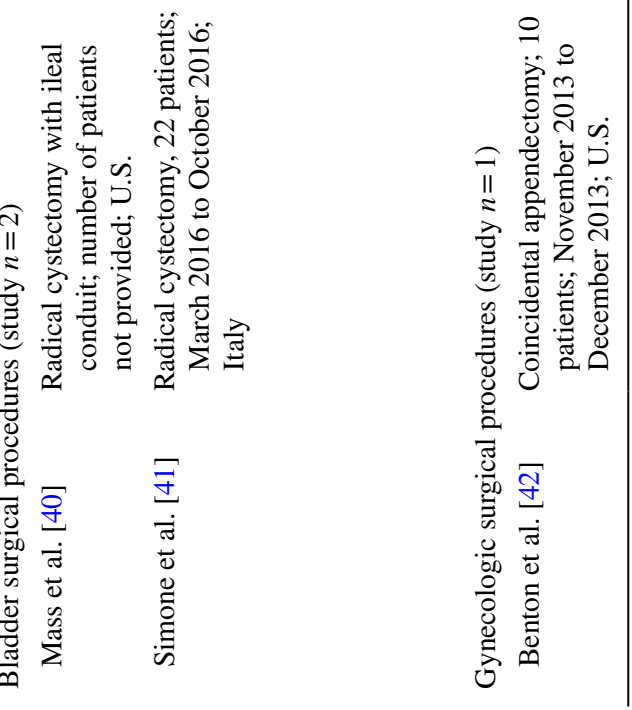


\section{Comparative assessments of da Vinci robotic surgical procedures performed with ELS vs. EWSS}

Only three recently conducted studies compared operative outcomes of robotic surgery with ELS vs. EWSS [7-9]. Hagen et al. compared 49 Roux-en-Y Gastric Bypass (RGB) surgeries performed with Echelon ELS with $60 \mathrm{~mm}$ reloads against $49 \mathrm{RGB}$ surgeries performed with the $45 \mathrm{~mm}$ EWSS (matching criteria: age, gender, body mass index) at the University Hospital Geneva [7]. Hagen also described technique details on the stapling job during gastric pouch formation and compared the costs associated with both stapling techniques.

Both groups were demographically similar and the authors reported unsuccessful clampings in $19 \%$ of all the recorded stapling attempts in the EWSS group $(n=211)$, requiring a wait time for staple firing and sometimes repositioning of the EWSS, which likely contributed to the 22-min difference of operative time between groups, in favor of Echelon ELS, although not statistically significant (216 min vs. $194 \mathrm{~min}, p=0.104$ ). No unsuccessful clampings were reported within the ELS group. The difference in stapler cartridge length (45 mm EWSS, $60 \mathrm{~mm}$ Echelon ELS) may have contributed to the significant difference in reloads used to create the gastric pouch, in favor of Echelon ELS $(4.1 \pm 1.1$ vs. $4.9 \pm 1.6, p=0.0048)$. Hence, there was a higher overall cost of stapling (\$2212 vs. $\$ 1787$ USD, $p<0.001$ ) in the EWSS group, not including the cost associated with longer operative time.

In a second study, Holzmacher et al. compared operative outcomes and stapler cost of robotic colorectal surgery (left, sigmoid, subtotal, and total colectomy; low anterior resection for malignancy, diverticular disease, or inflammatory bowel disease) performed using ELS with $45 \mathrm{~mm}$ reloads (manufacturer not specified) in 35 cases and EWSS with $45 \mathrm{~mm}$ reloads in 58 cases [8].

The groups were demographically similar, and the authors reported no significant differences in blood loss, operating times, hospital length of stay, or complication rates. There were more stapler firings in the ELS group (2.7 vs. 1.9 per patient), and the authors reported that the cost per patient for the ELS group was higher compared to the EWSS group ( $\$ 631$ vs. $\$ 473$ per patient, $p=0.001$ ). No patients in the ELS group required reoperations within 30 days, but three patients required reoperations in EWSS group $(p=0.05)$. On multivariate analysis, there was no statistically significant difference in the number of anastomotic leaks or overall complications between groups and the investigators of this study concluded that colorectal surgery performed with either EWSS or ELS are comparable in safety and effectiveness, but that EWSS may be more cost-effective than the $45 \mathrm{~mm}$ ELS in colorectal surgery.
In a third study, Atasoy et al. retrospectively compared operative outcomes and stapler utilization during robotic surgery for cancer performed with ELS with $60 \mathrm{~mm}$ reloads (Echelon Endopath, Ethicon; or Endo GIA Roticulator, Covidien) in 62 cases and with EWSS with $45 \mathrm{~mm}$ reloads in 45 cases [9].

The groups were demographically similar with the only exception being a greater percentage of male patients in the EWSS group (76 versus $55 \%, P=0.03$ ). The number of cartridges used were similar for both groups regardless of the type of stapler used in the procedure (ELS-2 vs. EWSS$2, P=0.58)$, and the overall complication rate was similar between the groups (ELS-24\% versus EWSS-31\%, $P=0.32$ ). Leak rates were also similar in both groups, 5 and $3 \%$ in the EWSS and ELS stapler groups, respectively $(p=1)$.

\section{Non-comparative assessments}

\section{Operative outcomes of da Vinci robotic surgical procedures performed with ELS}

Twenty-seven non-comparative studies reported on outcomes from da Vinci robotic surgeries performed with ELS; of these, 18 were with the Echelon ELS and 9 with the Endo GIA ELS. Robotic surgery performed with ELS is generally referred to as an advanced surgical technique for multiple types of procedures, including gastric bypass [10-12, 28], sleeve gastrectomy [12-15], liver resection [18, 19, 30, 31], colorectal surgery [20-24, 32-34], thoracic surgery [25], nephrectomy [26, 35], pancreatectomy [27], bladder surgery [34], and prostate surgery [36]. These procedures typically take longer than laparoscopic or open surgery [10, 11, 13, $14,18]$, but have comparable or lower complication rates and/or more favorable perioperative outcomes [11-14, 25, $27,36]$. In these studies, stapling was typically performed by an assistant laparoscopic surgeon, and it was generally reported that although technically demanding, surgical procedures performed with the da Vinci robotic system or ELS are practical and safe.

\section{Operative outcomes of da Vinci robotic surgical procedures performed with EndoWrist robotic staplers}

Six non-comparative studies (two manuscripts, four abstracts) reported on the use of the EWSS with the da Vinci robotic system. These initial experience reports generally suggested that totally robotic procedures, in bariatric [37], colorectal [38, 39], bladder [40, 41], and gynecological surgical procedures [42] may be safe and have the advantage of console surgeon autonomy and precise stapler control.

One of these non-comparative papers by Bae et al. described a single-case study of right-sided colon cancer where EWSS was used to create an intracorporeal 
anastomosis [38]. The reported case was successful and the surgeon performed stapling from the console. However, the authors cautioned against possible increased risk of inadvertent strictures caused by posterior bowel wall involvement during the intracorporeal stapling procedure, as well as increased operative time associated with intracorporeal anastomosis creation.

In another study, Benton et al. reported no intraoperative or postoperative complications in a case series of ten gynecologic surgeries where EWSS was used to complete coincidental appendectomies. However, the authors noted larger series of patients will be needed to evaluate safety and efficiency [42].

\section{Discussion}

A wide array of surgical procedures has been accomplished with the advanced technology of the da Vinci robotic system performed with either ELS or EWSS. Most studies in this systematic review are non-comparative reports of perioperative outcomes of robotic surgical procedures that used ELS for stapling jobs in robotic procedures; which is not surprising given the relatively recent (2014) launch of the EWSS. Although totally robotic surgical procedures may allow for the entire procedure to be completed by the console surgeon and no stapling-specific outcomes (e.g., staple line integrity, intraoperative misfires, and/or postoperative leakage) have yet been described in the literature, some studies in this systematic review suggest that there may be trade-offs to be considered when transitioning from using ELS for stapling jobs. A bariatric surgery, non-comparative series by Alper et al. reported that the EWSS staple load misfired in 3.3\% of patients who had revision bariatric surgeries [37]. This possible failure may align with issues mentioned in the FDA product recall database [6].

The study by Hagen et al. compared the $60 \mathrm{~mm}$ Echelon ELS (which is the most used reload size in bariatric procedures) vs. the only size $(45 \mathrm{~mm})$ reload offered with EWSS which explains, at least in part, the larger number of firings needed, and therefore cost, with the EWSS [7]. Hagen et al. also reported unsuccessful stapler clamping in nearly $20 \%$ of all recorded attempts with EWSS in bariatric surgery. The investigators suggest that the same design features, which confer the EWSS higher amounts of articulation, may also limit the clamping force of EWSS. The two other studies by Holzmacher et al. and Atasoy et al. did not report on stapler clamping failures in colorectal procedures $[8,9]$.

The study by Atasoy et al. compared the $45 \mathrm{~mm}$ EWSS vs. $60 \mathrm{~mm}$ ELS in rectal cancer surgery and found that the number of cartridges used were similar in both groups (costs were not compared in this study) [9]. Holzmacher et al., on the other hand, compared $45 \mathrm{~mm}$ EWSS vs. $45 \mathrm{~mm}$ ELS; which may not be reflective of the real-life preference for $60 \mathrm{~mm}$ ELS in many laparoscopic colorectal procedures [8]. Although the authors did not explain why fewer firings were needed with EWSS (if both groups used the same size reloads), this may explain, at least in part, the lower stapler cost in the EWSS group [8]. It is also difficult to assess if the difference in cost would have been the same with a different ELS brand, as there was no mention of the ELS brand used in their study.

Both the number of stapler firings required and tissue re-clamp rate, may impact a third criteria for consideration-operative time. Hagen et al. reported fewer stapler firings and a lower tissue re-clamp rate with Echelon ELS vs. EWSS, as well as shorter operative time with Echelon ELS vs. EWSS; however, given that the difference in operative time was not statistically significant [7], only studies with larger sample size may be able to corroborate this possible difference. Holzmacher et al. reported fewer firings with the $45 \mathrm{~mm}$ EWSS vs. $45 \mathrm{~mm}$ ELS, but the operative time was similar between groups ( 270 vs. $264 \mathrm{~min}, p=0.769$ ) [8].

When evaluating the cost of ELS and EWSS, Hagen et al. found EWSS stapling costs to be significantly higher than Echelon ELS for bariatric procedures (2212 vs. 1787 USD, $p=0.0001$ ) [7]. On the other hand, Holzmacher et al. found ELS (manufacturer not reported) stapling costs to be significantly higher than EWSS for colorectal procedures (631 vs. 473 USD, $p=0.001$ ) [8]. Atasoy et al. did not report stapler costs [9]. The cost structures for ELS and EWSS are different and must be carefully considered when evaluating device value. For the cost assessment of EWSS, the cost per fire can be determined by adding the cost of the stapling device, which is reusable up to 50 uses, with the stapler reloads [7]. The cost calculation for EWSS should also consider the use of trocar reducers, cannula seals, and stapler sheaths that are necessary to operate the device. For ELS, on the other hand, the cost per fire should be calculated with consideration for the acquisition of only the stapling device and reloads.

\section{Conclusions}

Systematic reviews like this one are at best able to offer insights, formulate new hypotheses to test, and ascertain the status of a subject or procedure. They are not able to draw firm conclusions or make clear recommendations because of the limited number of comparative reports, coupled with the small sample sizes, and the heterogeneity of the surgical procedures involved in the studies evaluated. The key finding in this literature review is that there is very little comparative perioperative data between the use of ELS and EWSS in robotic surgery (three studies). Given that ELS has a longer clinical history and relatively more robust evidence base (ELS-27 studies; EWSS-6 studies), surgeons and medical 
device purchasers should consider possible trade-offs before switching their entire clinical utilization to EWSS.

Author contributions The authors would like to thank Jay Lin and Melissa Smith (Novosys Health) for medical writing assistance. All authors contributed equally. All authors read and approved the final manuscript.

Funding This research and preparation of this manuscript was supported by Ethicon, a Johnson \& Johnson company.

\section{Compliance with ethical standards}

Conflict of interest Mario Gutierrez is an employee of Ethicon, a Johnson \& Johnson company. Richard Ditto is an employee of Ethicon, a Johnson \& Johnson company. Sanjoy Roy is an employee of Ethicon, a Johnson \& Johnson company.

Open Access This article is distributed under the terms of the Creative Commons Attribution 4.0 International License (http://creativeco mmons.org/licenses/by/4.0/), which permits unrestricted use, distribution, and reproduction in any medium, provided you give appropriate credit to the original author(s) and the source, provide a link to the Creative Commons license, and indicate if changes were made.

\section{References}

1. Chekan E, Whelan R (2014). Surgical stapling device-tissue interactions: what surgeons need to know to improve patient outcomes. Med Dev Evid Res. https://doi.org/10.2147/mder.s67338

2. Griffith PS, Birch D, Sharma A, Karmali S (2012) Managing complications associated with laparoscopic Roux-en-Y gastric bypass for morbid obesity. Can J Surg 55(5):329-336. https:// doi.org/10.1503/cjs.002011

3. El-Kadre L, Tinoco AC, Tinoco RC, Aguiar L, Santos T (2013) Overcoming the learning curve of laparoscopic Roux-en-Y gastric bypass: a 12-year experience. Surg Obes Relat Dis 9(6):867-872. https://doi.org/10.1016/j.soard.2013.01.020

4. Thuler FR, Freitas WR, Ilias EJ, Kassab P, Malheiros CA (2014). Laparoscopic bariatric surgery training program model: gastric bypass. BMC Surg. https://doi.org/10.1186/1471-2482-14-101

5. Lublin M, Lyass S, Lahmann B, Cunneen SA, Khalili TM, Elashoff JD, Phillips EH (2005) Leveling the learning curve for laparoscopic bariatric surgery. Surg Endosc 19(6):845-848. https ://doi.org/10.1007/s00464-004-8201-x

6. U.S. Food \& Drug Administration. Medical Device Recalls. Updated 7/29/2017. https://www.accessdata.fda.gov/scripts/cdrh/ cfdocs/cfRES/res.cfm?start_search=1\&event_id=\&productdes criptiontxt $=$ EndoWrist $\&$ productcode $=\&$ IVDProducts $=\&$ rootC auseText $=\&$ recallstatus $=\&$ centerclassificationtypetext $=\&$ recal lnumber $=\&$ postdatefrom $=\&$ postdateto $=\&$ productshortreasont $x$ $\mathrm{t}=\&$ firmlegalnam $=\& P M A \_510 \mathrm{~K} \_N u m=\&$ pnumber $=\& \mathrm{knumb}$ er $=\&$ pagenum $=10 \&$ sortcolumn=cddd. Accessed 15 Sept 2017

7. Hagen ME, Jung MK, Fakhro J, Buchs NC, Buehler L, Mendoza JM, Morel P (2017). Robotic versus laparoscopic stapling during robotic Roux-en-Y gastric bypass surgery: a case-matched analysis of costs and clinical outcomes. Surg Endosc. https://doi. org/10.1007/s00464-017-5707-6

8. Holzmacher JL, Luka S, Aziz M, Amdur RL, Agarwal S, Obias V (2017) The use of robotic and laparoscopic surgical stapling devices during minimally invasive colon and rectal surgery: a comparison. J Laparoendosc Adv Surg Techn 27:151-155

9. Atasoy D, Aytac E, Ozben V, Bayraktar O, Bayraktar IE, Aghayeva A et al (2018) Robotic versus laparoscopic stapler use for rectal transection in robotic surgery for cancer. J Laparoendosc Adv Surg Tech 28(5):501-505

10. Smeenk RM, van't Hof G, Elsten E, Feskens PGBM (2016) The results of 100 robotic versus 100 laparoscopic gastric bypass procedures: a single high volume centre experience. Obes Surg 26:1266-1273

11. Myers SR, McGuirl J, Wang J (2013) Robot-assisted versus laparoscopic gastric bypass: comparison of short-term outcomes. Obes Surg 23:467-473

12. Kosanovic R, Romero RJ, Donkor C (2015) A comparative retrospective study of robotic sleeve gastrectomy vs robotic gastric bypass. Int J Med Robot 11:275-283

13. Ijah RF, Bhatia P, Kaltan S, Khetan M, John S, Bindal V, Ali A (2014) Sleeve gastrectomy for morbid obesity: robotic vs standard laparoscopic sleeve gastrectomy methods. World J Laparosc Surg 7:1-6

14. Vilallonga R, Fort JM, Caubet E, Gonzalez O, Armengol M (2013) Robotic sleeve gastrectomy versus laparoscopic sleeve gastrectomy: a comparative study with 200 patients. Obes Surg 23:1501-1507

15. Diamantis T, Alexandrou A, Nikiteas N, Giannopoulos A, Papalambros E (2011) Initial experience with robotic sleeve gastrectomy for morbid obesity. Obes Surg 21:1172-1179

16. Vilallonga R, Fort JM, Caubet E (2015). Robotically assisted single anastomosis duodenoileal bypass after previous sleeve gastrectomy implementing high valuable technology for complex procedures. J Obes 2015:586419

17. Dogra PN, Regmi SK, Singh P, Bora G, Saini AK, Aggarwal S (2014) Robot-assisted laparoscopic augmentation ileocystoplasty in a tubercular bladder. Urol Ann 6:152-155

18. Xu Y, Wang H, Ji W, Tang M, Li H, Leng J, Meng X, Dong J (2016) Robotic radical resection for hilar cholangiocarcinoma: perioperative and long-term outcomes of an initial series. Surg Endosc 30:3060-3070

19. Calin ML, Sadiq A, Arevalo G, Fuentes R, Flanders VL, Gupta N, Nasri B, Singh K (2016) The first case report of robotic multivisceral resection for synchronous liver metastasis from pancreatic neuroendocrine tumor: a case report and literature review. J Laparoendosc Adv Surg Tech A 26:816-824

20. Morelli L, Guadagni S, Di Franco G, Palmeri M, Caprilli G, D'isidoro C, Pisano R, Marciano E, Moglia A, Di Candio G, Mosca F (2016) Short-term clinical outcomes of robot-assisted intersphincteric resection and low rectal resection with doublestapling technique for cancer: a case-matched study. Int J Colorectal Dis 31:737-739

21. Bae SU, Jeong WK, Bae OS, Baek SK (2016) Reduced-port robotic anterior resection for left-sided colon cancer using the Da Vinci single-site platform. Int J Med Robot 12:517-523

22. Morelli L, Perutelli A, Palmeri M (2016) Robot-assisted surgery for the radical treatment of deep infiltrating endometriosis with colorectal involvement: short- and mid-term surgical and functional outcomes. Int J Colorectal Dis 31:643-652

23. Leong QM, Son DN, Cho JS, Amar AH, Kim SH (2012) Robotassisted low anterior resection for situs inversus totalis: a novel technical approach for an uncommon condition. Surg Laparosc Endosc Percutan Tech 22:e87-e90

24. Trastulli S, Desiderio J, Farinacci F, Ricci F, Listorti C, Cirocchi R, Boselli C, Noya G, Parisi A (2013) Robotic right colectomy for cancer with intracorporeal anastomosis: short-term outcomes from a single institution. Int J Colorectal Dis 28:807-814 
25. Rinieri P, Peillon C, Salaün M, Mahieu J, Bubenheim M, Baste JM (2016) Perioperative outcomes of video- and robot-assisted segmentectomies. Asian Cardiovasc Thorac Ann 24:145-151

26. Giacomoni A, Di Sandro S, Lauterio A (2013) Initial experience with robot-assisted nephrectomy for living-donor kidney transplantation: feasibility and technical notes. Transpl Proc 45:2627-2631

27. Liu R, Liu Q, Zhao Z, Tan XL, Gao YX, Zhao GD (2017) Robotic vs. laparoscopic distal pancreatectomy: a propensity score-match study. J Surg Oncol 116:461-469

28. Reche F, Mancini A, Borel AL, Faucheron JL (2016) Totally robotic reversal of omega-loop gastric bypass to normal anatomy. Obes Surg 26:1994-1995

29. Vasilescu C, Procopiuc L (2012) Robotic surgery of locally advanced gastric cancer: a single-surgeon experience of 41 cases. Chirurgia (Bucur) 107:510-517

30. Montalti R, Scuderi V, Patriti A, Vivarelli M, Troisi RI (2016) Robotic versus laparoscopic resections of posterosuperior segments of the liver: a propensity score-matched comparison. Surg Endosc 30:1004-1013

31. Salloum C, Tayar C, Laurent A, Malek A, Compagnon P, Memeo R, De Angelis N, Pascal G, Azoulay D (2014) Robot-assisted laparoscopic hepatectomy: the henri-mondor experience. In: 11th world congress of the International Hepato-Pancreato-Biliary association. Seoul South Korea (abstract)

32. Ahmed J, Nasir M, Flashman K, Khan J, Parvaiz A (2016) Totally robotic rectal resection: an experience of the first 100 consecutive cases. Int J Colorectal Dis 31:869-876

33. Roviello F, Piagnerelli R, Ferrara F, Scheiterle M, De Franco L, Marrelli D (2015) Robotic single docking total colectomy for ulcerative colitis: first experience with a novel technique. Int J Surg 21:63-67

34. Liu N, Risk M, George V, Robb B, Gardner T (2012) Incisionless dual diversions: creation of urostomy and colostomy using the da Vinci robot. Annual meeting of the American Urological
Association, AUA. Atlanta, GA United States. J Urol 4(Suppl 1):e356 (abstract)

35. Patel MN, Aboumohamed A, Hemal A (2015) Does transition from the da Vinci Si to Xi robotic platform impact single-docking technique for robot-assisted laparoscopic nephroureterectomy? BJU Int 116:990-994

36. Wit EMK, De Jong J, Acar C, VAN Muilekom E, Tillier C, De Blok W, Poel VANDER. H (2016) Stapling for prostate pedicle management during robot-assisted radical prostatectomy. Minerva Urol Nefrol 68:429-436

37. Alper N, Bassiri-Tehrani B, Marks Y, Taggart J, Teixeira MD (2017) The safety and efficacy of the Da Vinci robotic stapler in robotic-assisted bariatric surgery. Surg Endosc 31:S190-S259 (abstract)

38. Bae SU, Jeong WK, Baek SK (2016) Single-port plus an additional port robotic complete mesocolic excision and intracorporeal anastomosis using a robotic stapler for right-sided colon cancer. Ann Surg Treat Res 91:212-217

39. Guadagni S, di Franco G, Palmeri M, Furbetta N, Bianchinni M, Gianardi D, Lucchesi M, Stefanini G, Caprili G, d'Isodoro C, Melfi F, di Candio G, Mosca F, Morelli L (2017) New technologies in robotic rectal resection: da Vinci Xi, Integrated Table Motion, Endowrist Robotic Stapler and indocyanine green fluorescence. Surg Endosc 31:S190-S259 (abstract)

40. Mass AY, Zhao LC, Huang WC (2014) Robotic intracorporeal ileal conduit using the intuitive Endowrist one robotic stapler. J Endourol 28(suppl 1):A314 (abstract)

41. Simone G, Guaglianone S, Minisola F, Ferriero M, Misuraca L, Tuderti G, Romeo G, Gallucci M (2017) Intracorporeal partly stapled Padua ileal bladder using robotic staplers: surgical technique, perioperative and early functional outcomes of a prospective single center series. J Urol 197(suppl):e681 (abstract)

42. Benton SA, Riley KA, Harkins GJ (2015) The use of a robotic stapling device for coincidental appendectomy. J Gynecol Surg $31: 148-150$ 